\title{
Electron Spectrum of the Dragonfly Pulsar Wind Nebula from X-ray to TeV
}

\author{
Chad Brisbois* \\ Michigan Technological University, Houghton, Michigan, USA \\ E-mail: cbrisboiemtu.edu
}

Vikas Joshi

Friedrich-Alexander-Universität Erlangen-Nürnberg, Erlangen Centre for Astroparticle Physics, Erlangen, Germany

E-mail: vikas.joshi@fau.de

\section{For the HAWC Collaboration}

Full author list and acknowledgements:

https: / / www.hawc-observatory •org/collaboration/icrc2019.php

See also PoS(ICRC2019)1177

The Dragonfly nebula is a Vela-like Pulsar Wind Nebula (PWN) in the Cygnus region powered by the spin down of PSR J2021+3651. In X-rays, the inner nebula is a few arcseconds across, whereas at TeV energies VERITAS and HAWC has observed extended emission much larger than the extension in X-rays. The TeV gamma-ray source HAWC J2019+368 was originally discovered in 2007 by the Milagro Observatory and has been associated with this pulsar. Recent work has shown hints of energy dependent morphology for the source at TeV energies, supporting the interpretation of the gamma-ray emission being due to Inverse Compton scattering of electrons and positrons from interstellar radiation fields and cosmic microwave background photons. The hard spectral index and spectral softening above $30 \mathrm{TeV}$ of the source are consistent with KleinNishina suppression of the electron-photon cross-section at high energies. We will present our most recent studies in modeling and analysis of HAWC J2019+368, explaining the X-ray and $\mathrm{TeV}$ emission up to the highest energies using the latest data from the HAWC Observatory.

36th International Cosmic Ray Conference -ICRC2019-

July 24th - August 1st, 2019

Madison, WI, U.S.A.

\footnotetext{
${ }^{*}$ Speaker.
} 


\section{Introduction}

PSR J2021+3651 was originally discovered in 2002 as a followup to an EGRET source [1]. Soon after, its X-ray PWN was discovered and found to have a double torus structure, a feature only previously found in the Vela PWN [2,3]. Milagro first observed extended TeV emission in this region, tentatively associating MGRO J2019+37 with PSR J2021+3651, but the measured morphology was too extended and angular resolution too wide to make firm conclusions [4]. VERITAS observed this region and discovered two sources, VER J2019+368 and VER J2016+371 [5, 6]. VER J2019+368 is coincident with PSR J2021+3651 and Sh 2-104 (an HII region), although followup observations show that Sh 2-104 is unlikely to contribute significantly to the observed TeV emission [7]. Two publications by the VERITAS collaboration show that VER J2019+368 has an extended, asymmetric morphology [5, 6]. The VERITAS collaboration has reported a power law energy spectrum up to $\sim 40 \mathrm{TeV}$ for VER J2019+368, with spectral softening at higher energies not being significantly preferred [6]. Previous publications by the VERITAS Collaboration have associated VER J2016+371 with the supernova remnant CTB 87, although it is also possible that there is some contribution from the BL Lac object QSO J2015+371[6]. The ARGO-YBJ experiment saw no significant excess coincident with VER J2016+371 [8]. The HAWC Collaboration has recently developed energy estimation techniques, which allow for the measurement of $\gamma$-ray energies in excess of $100 \mathrm{TeV}$ [9]. HAWC J2019+368 is currently one of the only detected sources in the sky with emitting significant flux in $\gamma$-rays above $100 \mathrm{TeV}$ [10]. Understanding the physical processes giving rise to this $\gamma$-ray emission is important to understanding the limits of particle acceleration in pulsar powered systems.

\section{Analysis and Description of the Region}

Originally reported as a single source $2 \mathrm{HWC}$ J2019+367[11], this dedicated analysis has identified two sources, HAWC J2019+368 and HAWC J2016+371. A significance map of the region assuming an $\alpha=-2.7$ spectrum with the nearby TeVCat sources and ROI labeled can be seen in Figure 1. The dataset analyzed included 1038 days of data using the ground parameter energy estimator (more details in [9]).

The analysis was performed in a $3^{\circ}$ Region Of Interest (ROI) centered at RA, Dec $=304.8690^{\circ}$, $36.7710^{\circ}$. The fit was performed using the hawc_hal plugin for 3ML [12]. The nominal model consists of two physical sources, and one background source which accounts for sources leaking into the ROI. HAWC J2019+368 is the primary focus of this work, and HAWC J2016+371 was found in the course of the analysis. HAWC J2019+368 is modeled as an asymmetric Gaussian source with a log parabola energy spectrum. HAWC J2016+371 is modeled as a point source with a power law energy spectrum. The optimized morphological parameters for HAWC J2019+368 are similar to what the VERITAS collaboration has reported [5, 6]. The energy spectrum of HAWC J2019+368 is given in Figure 4. We scale the flux measured by VERITAS [6] up to the size of the morphology measured by VERITAS, rather than the extraction region of $0.23^{\circ}$ in Figure 4.

Prior to its inclusion in this analysis, HAWC J2016+371 was first seen as significant excess in the residual map after subtracting HAWC J2019+368. Its inclusion in the model significantly 


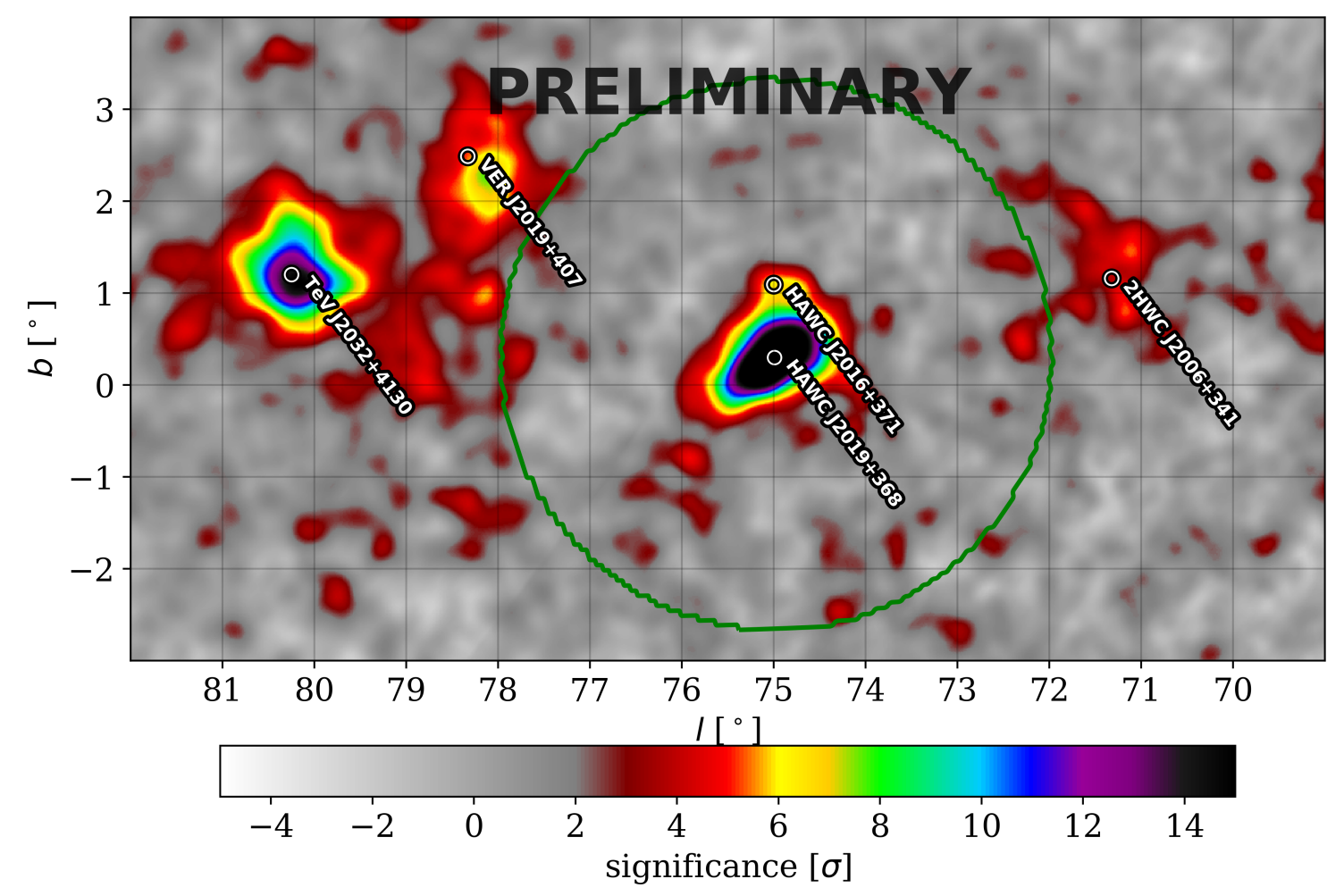

Figure 1: Significance map of the Cygnus region as seen by HAWC with TeV sources labeled. This point source map is produced by fitting the flux normalization to a power law spectrum with a fixed index of $\alpha=-2.7$ at each pixel on the sky. The ROI for the analysis detailed here is indicated by the green contour.

improved the description of the ROI $(\triangle T S=35)$. After including the uniform background, the significance is $T S=27$ and $T S=984$ for HAWC J2016+371 and HAWC J2019+368, respectively.

\subsection{Energy Dependent Morphology}

We searched for energy dependent morphology in the HAWC data. Energy dependent morphology is a signature of leptonic origin for $\gamma$-ray emission, because of electrons relatively fast cooling time relative to hadrons [13]. Considering longitudinal profiles along the major axis of HAWC J2019+368, there are hints of decreasing extent with increasing energy (See Figure 3). The profiles can be seen in Figure 2, using the position of PSR J2021+3651 as the zero point. In Figure 3 , we see a trend of decreasing size with increasing energy, but it is not statistically significant.

\section{Modeling of Electron spectrum}

Assuming the $\gamma$-ray emission is due to inverse compton scattering, we use the naima package $[14,15]$. The electron spectrum was assumed to follow a broken power law with an exponential cutoff following the work in [16]. We fit the normalization and cutoff energies, but left the indices (2.1 and -3.1$)$ and break energy $(80 \mathrm{TeV})$ fixed to maintain consistency with the X-ray measurements from Suzaku [16]. The inverse Compton (IC) band in Figure 4 represents the $68 \%$ containment 


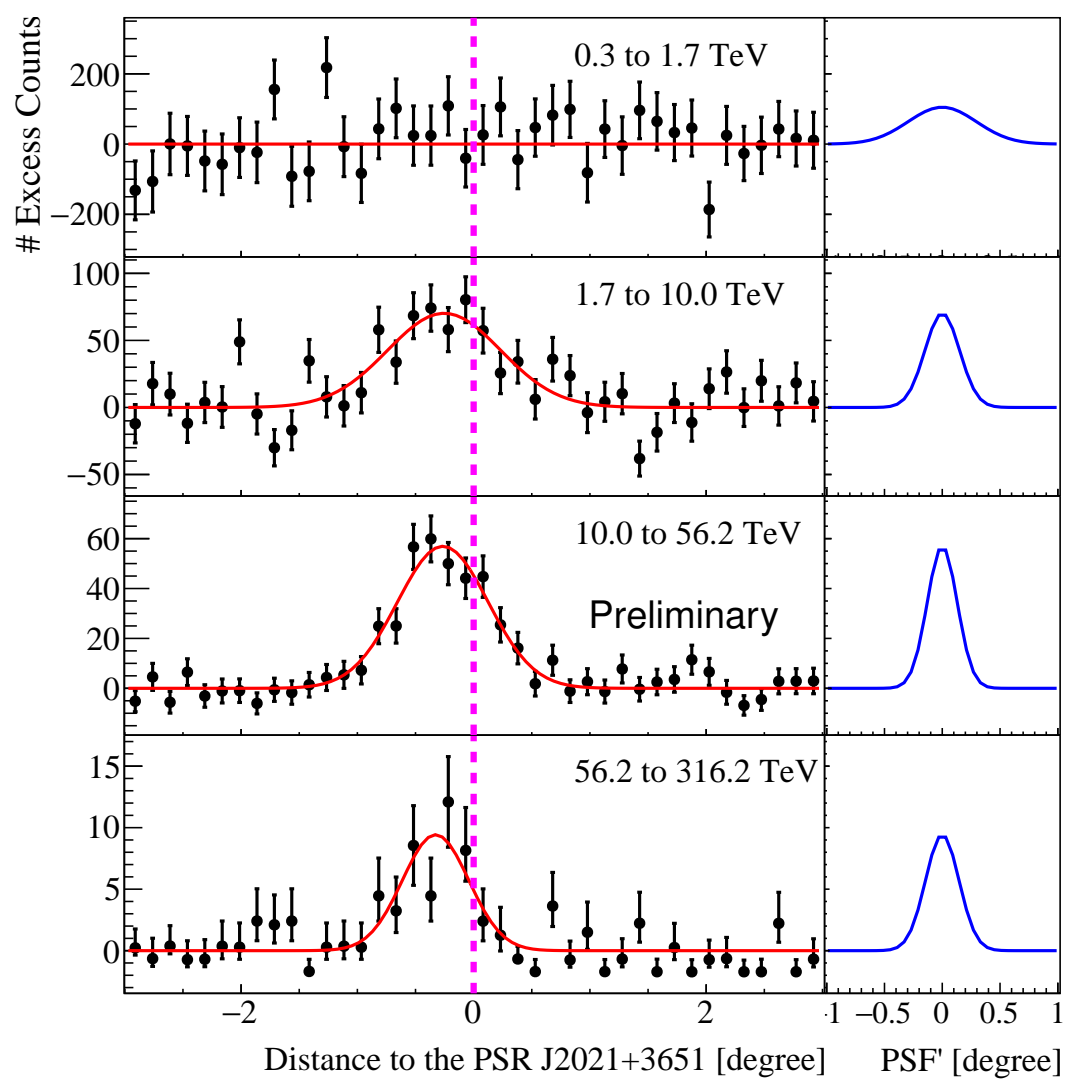

Figure 2: Longitudinal profile plot along the major axis of HAWC J2019+368. The profile is $0.7^{\circ} \times 6^{\circ}$ with 40 bins. The Point Spread Function (PSF') in each energy band is given on the right side in blue, for comparison to the fit in red on the profile. It is the $\operatorname{width}(\sigma)$ of a 1D Gaussian from the longitudinal profile of that point source about an arbitrary axis. It is obtained from a simulated point source with a spectral index of -2.2 . Note the different scales for excess counts in each energy band.

region of the inverse Compton spectrum for the present-day electron spectrum to HAWC data. The interstellar radiation fields included were the Cosmic Microwave Background and a $30 \mathrm{~K}$ blackbody field with an energy density of $0.3 \mathrm{eV} \mathrm{cm}^{-3}$. Alternative spectral models for the present day electron spectrum are under study. This fit also allows for the calculation of the energy in the electrons (calculated above $1 \mathrm{MeV}$ ) emitted by PSR J2021+3651. The total energy of the electrons in the spectrum predicts $\sim 18 \%$ of the pulsar's spindown power has gone into the production of electrons and positrons under the assumption of constant $\dot{E}$ over the characteristic age of the pulsar. Accounting for time dependence will decrease this fraction.

\section{Discussion}

This work is the first detailed multi-source analysis using HAWC data up to the highest energies of the source region containing 2HWC J2019+367. The morphology for HAWC J2019+368 


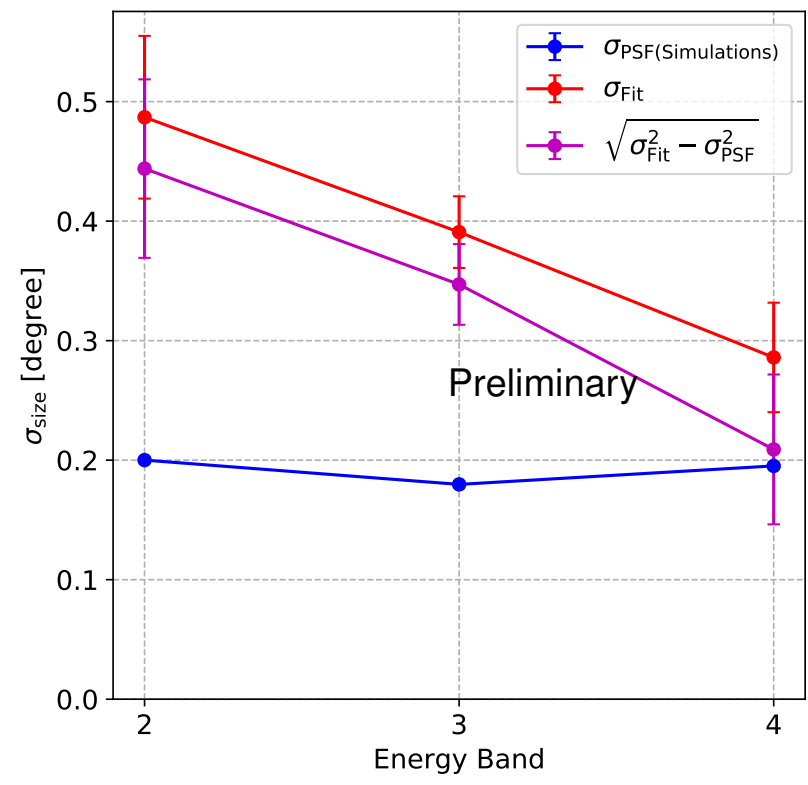

Figure 3: The measured width compared to the PSF in each energy band from Figure 2. A trend is seen, but given the number of data points and uncertainties, it is not statistically significant. The width of the PSF is plotted in blue.

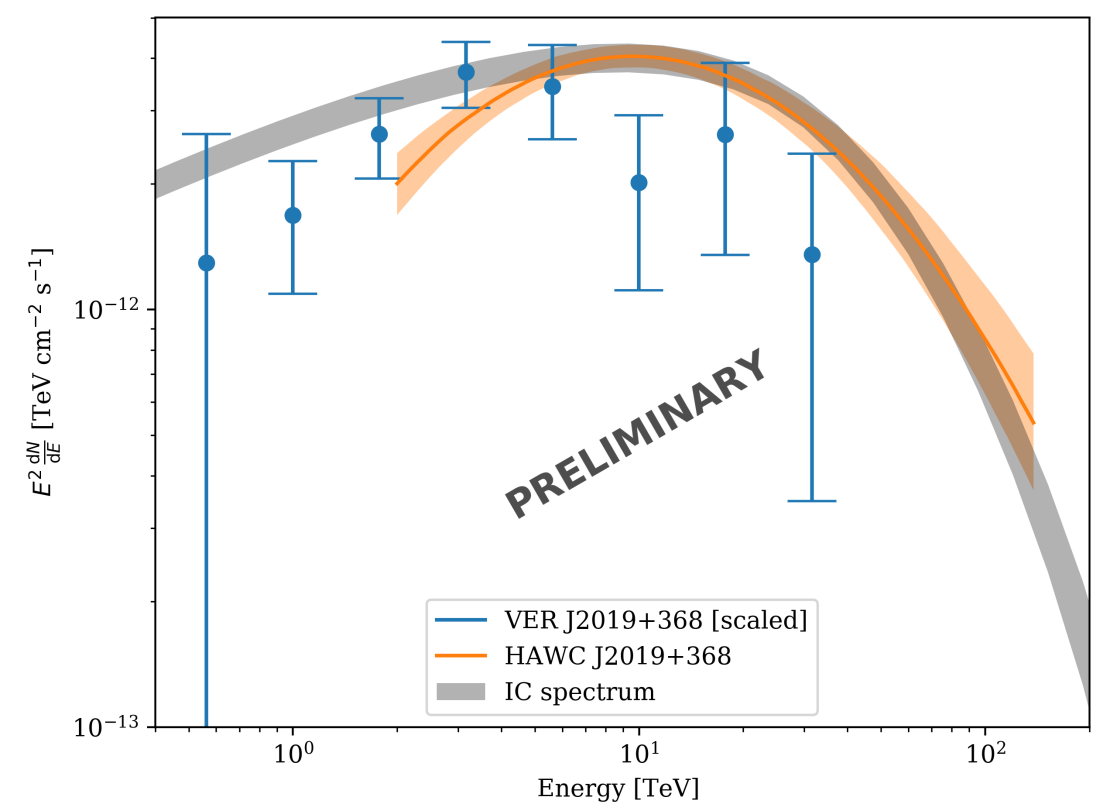

Figure 4: The spectral energy distribution of HAWC J2019+368 measured by HAWC in the latest data set. VERITAS flux points are taken from [6]. The grey band represents the $68 \%$ containment for the inverse Compton (IC) spectrum obtained from naima [15] using the same functional form as [16]. 


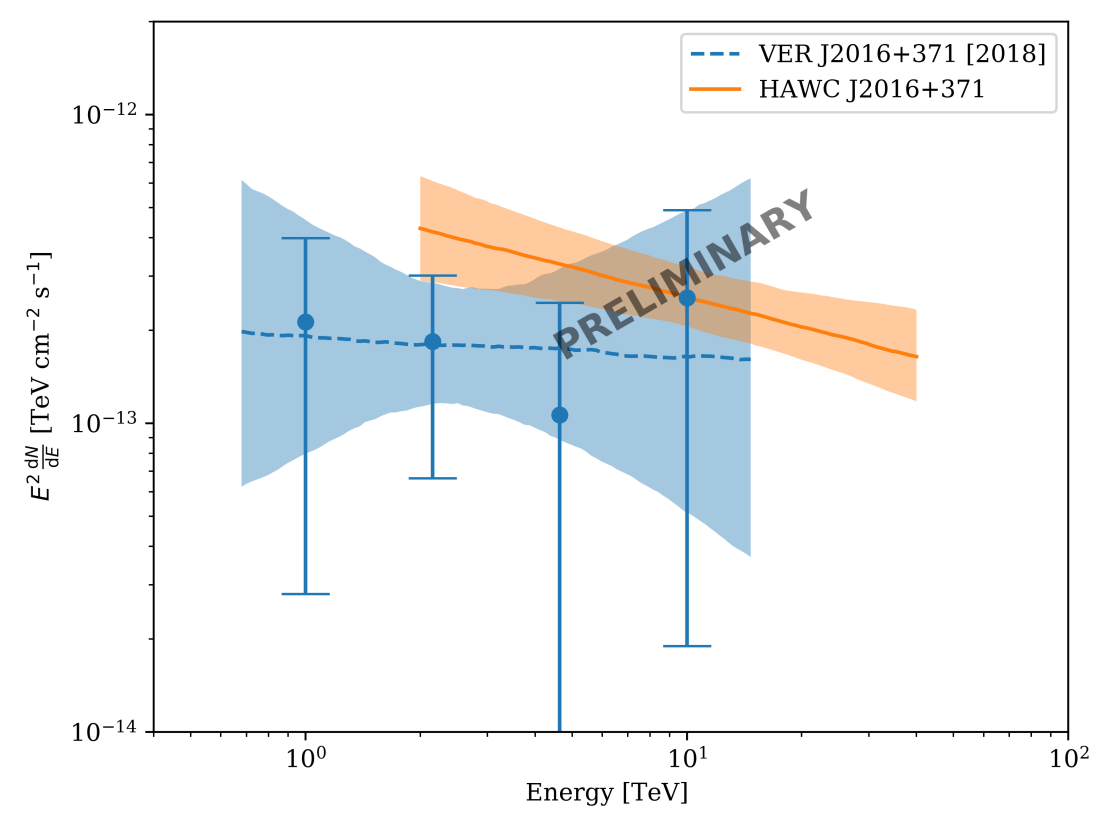

Figure 5: The spectral energy distribution of HAWC J2016+371 measured by HAWC. Both sources are modeled as point sources in their respective analysis, and no scaling of the flux is performed. VERITAS flux points and fit taken from [6].

is found to be very similar to VER J2019+368, with an asymmetric Gaussian morphology being significantly preferred over symmetric models. The spectrum for HAWC J2019+368 agrees with the scaled VERITAS measurements, confirming that the result is consistent with previously published results. This is the highest energy flux measurements ever observed from this region of the sky, and this emission appears consistent with a model of electrons developed to explain X-ray and $\mathrm{TeV}$ data from Suzaku and VERITAS [7]. We are working on an $a b$ initio $\gamma$-ray spectrum from an electron source using GAMERA [17] and a more detailed interstellar radiation field.

This study is the first work to observe a TeV source coincident with VER J2016+371. Assuming that VER J2016+371 and HAWC J2016+371 are the same source (positional difference of $0.09^{\circ}$, within uncertainties), the spectrum has been measured to extend up to tens of $\mathrm{TeV}$, with similar flux and index as reported by VERITAS [6]. More data will be required to test for curvature in the spectrum at higher energies. The new techniques developed in [9], combined with HAWC's unparalleled uptime will allow for new multi-source studies of the form presented here to be performed in other areas of the sky, potentially leading to many new discoveries. 


\section{References}

[1] M. S. E. Roberts, J. W. T. Hessels, S. M. Ransom, et al., Astrophys. J. 577 (2002) L19-L22.

[2] J. W. T. Hessels, M. S. E. Roberts, S. M. Ransom, et al., Astrophys. J. 612 (2004) 389-397.

[3] A. Van Etten, R. W. Romani, and C. Ng, Astrophys. J. 680 (2008) 1417-1425.

[4] A. A. Abdo, U. Abeysekara, B. T. Allen, et al., Astrophys. J. 753 (2012) 159.

[5] E. Aliu, T. Aune, B. Behera, et al., Astrophys. J. 788 (2014) 78.

[6] A. U. Abeysekara, A. Archer, T. Aune, et al., Astrophys. J. 861 (2018) 134.

[7] E. V. Gotthelf, K. Mori, E. Aliu, et al., Astrophys. J. 826 (2016) 25.

[8] B. Bartoli, P. Bernardini, X. J. Bi, et al., Astrophys. J. 745 (2012) L22.

[9] HAWC Collaboration, A. U. Abeysekara, A. Albert, et al., arXiv e-prints (2019) arXiv:1905.12518.

[10] K. Malone, PoS ( ICRC2019) 734 (2019).

[11] A. U. Abeysekara, A. Albert, R. Alfaro, et al., Astrophys. J. 843 (2017) 40.

[12] G. Vianello, R. J. Lauer, P. Younk, et al., arXiv:1507.08343.

[13] J. Hinton and W. Hofmann, Annu. Rev. Astron. Astrophys. 47 (2009) 523-565.

[14] D. Khangulyan, F. A. Aharonian, and S. R. Kelner, Astrophys. J. 783 (2014) 100.

[15] V. Zabalza, Naima: a Python package for inference of particle distribution properties from nonthermal spectra, in 34th Int. Cosm. Ray Conf., p. 922, 2015.

[16] T. Mizuno, N. Tanaka, H. Takahashi, et al., Astrophys. J. 841 (2017) 104.

[17] J. Hahn, GAMERA - a new modeling package for non-thermal spectral modeling, in 34th International Cosmic Ray Conference (ICRC2015), vol. 34 of International Cosmic Ray Conference, p. $917,2015$. 